\title{
Systematic Discovery of New Genes in the Saccharomyces cerevisiae Genome
}

\author{
Marco M. Kessler, Qiandong Zeng, Sarah Hogan, Robin Cook, Arturo J. Morales, ${ }^{1}$ \\ and Guillaume Cottarel
}

Genome Therapeutics Corporation, Waltham, Massachusetts 02453, USA

\begin{abstract}
We used genome-wide comparative analysis of predicted protein sequences to identify many novel small genes, named smORFs for small open reading frames, within the budding yeast genome. Further analysis of 117 of these new genes showed that $8 \overline{4}$ are transcribed. We extended our analysis of one smORF conserved from yeast to human. This investigation provides an updated and comprehensive annotation of the yeast genome, validates additional concepts in the study of genomes in silico, and increases the expected numbers of coding sequences in a genome with the corresponding impact on future functional genomics and proteomics studies.
\end{abstract}

Current consensus suggests the number of yeast genes to be very close to 6000 (Goffeau et al. 1996; Mewes et al. 1997; Winzeler and Davis 1997). The number of protein-coding genes with open reading frames (ORFs) longer than 100 codons is predicted to be between 5300 and 5400 (Mackiewicz et al. 2002). Two recent studies suggest that the determination of gene numbers by stringent gene identification methods may underestimate the number of genes in human and other organisms (Gopal et al. 2001; Reboul et al. 2001). The initial annotation of the budding yeast genome did not include many short open reading frames (ORFs; Andrade et al. 1997; Olivas et al. 1997). Recent experimental studies designed to catalog all genome transcripts using SAGE technology (Velculescu et al. 1997; Basrai et al. 1999) and the analysis of a collection of transposon insertions (Ross-Macdonald et al. 1999) have discovered new ORFs that were not previously identified in silico. This pool of genes includes some that code for putative proteins that are shorter than 100 amino acids (Velculescu et al. 1997; Basrai et al. 1999; Ross-Macdonald et al. 1999). Two recent studies described the use of comparative sequence analysis between the genome of Saccharomyces cerevisiae and those of other hemiascomycetous yeasts (Blandin et al. 2000) or other Saccharomyces genomes (Cliften et al. 2001) to discover small nonannotated protein-coding and nonprotein-coding genes in chromosomal regions previously considered intergenic. In addition, transposon insertion was recently used to identify yeast genes that were previously overlooked (Kumar et al. 2002).

Here we describe a systematic in silico method to identify new small genes in $S$. cerevisiae that is an extension of the searches conducted by Blandin and Cliften (Blandin et al. 2000; Cliften et al. 2001) by using a more comprehensive database of fungal sequences. In addition we provide a comprehensive demonstration that the majority of the genes predicted are actually transcribed. Our findings from comprehensive database searches and experimental studies suggest that the number of coding genes in S. cerevisiae is substantially higher than currently believed.

'Corresponding author.

E-MAIL arturo.morales@genomecorp.com; FAX (781) 398-2476. Article and publication are at http://www.genome.org/cgi/doi/10.1101/ gr.232903.

\section{RESULTS}

\section{Identification of New ORFs}

While comparing whole fungal genomes, we (Zeng et al. 2001) as well as others (Kupfer et al. 1997; Tzung et al. 2001) made the observation that fungal translated ORFeomes are very diverse. For example, many $S$. cerevisiae proteins do not have homologs in Candida albicans (Tzung et al. 2001). However, comparison of $S$. cerevisiae predicted translated ORFs with a comprehensive fungal database that includes predicted protein sequences from C. albicans, Schizosacharomyces pombe, Aspergillus nidulans and fumigatus, Cryptococcus neoformans, Fusarium sporotrichioides, Neurospora crassa, and Pneumocystis carinii suggests that most budding yeast translated ORFs have homologs in one or more other fungal genomes (Zeng et al. 2001). We used this observation to identify novel proteincoding sequences in the budding yeast genome.

Our approach to identify candidate ORFs for new genes in the $S$. cerevisiae genome is outlined in Figure 1. Briefly, we

\section{smORFs Identification}

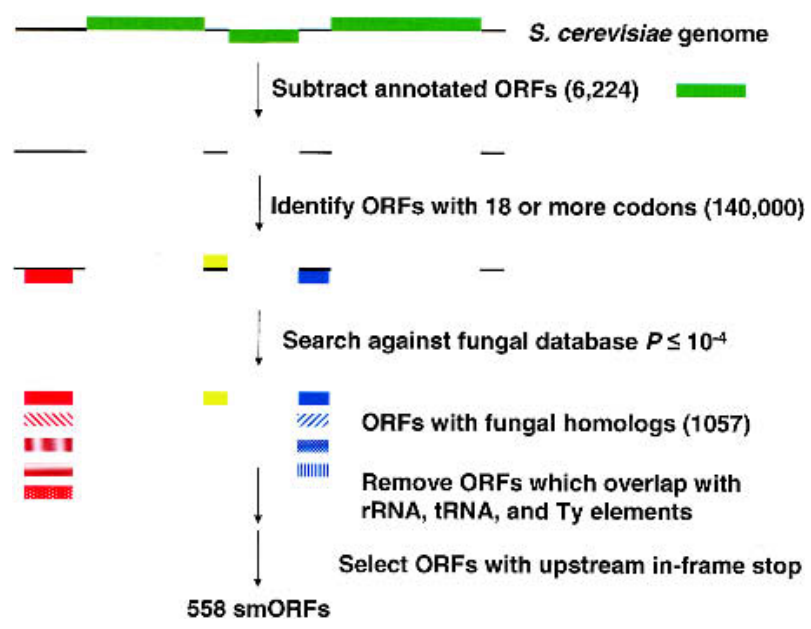

Figure 1 Overall strategy for smORF identification. Computational method used to identify new ORFs not identified by conventional methods. 
Table 1. List of S. cerevisiae smORFs for Which a Transcript was Detected

\begin{tabular}{|c|c|c|c|c|c|c|c|c|}
\hline smorf & $\begin{array}{c}\text { Original } \\
\text { name }\end{array}$ & Chromososme & Coordinates & $\begin{array}{l}\text { Size } \\
\text { (aa) }\end{array}$ & CAI & $\begin{array}{c}\text { RT-PCR } \\
\text { product (bp) }\end{array}$ & $\begin{array}{c}\text { SGD } \\
\text { annotation }\end{array}$ & Notes \\
\hline 3 & SR13 & chr1 & $124491-124306$ & 62 & 0.243 & 39 & & \\
\hline 13 & & chr2 & 18437-18177 & 87 & 0.181 & 99 & & \\
\hline $18^{a}$ & smORF2 & chr2 & 89973-90221 & 82 & 0.154 & 205 & YBL071W-A & \\
\hline $19^{c}$ & SR12 & chr2 & $91789-92025$ & 79 & 0.241 & 200 & YBL069W & embedded ORF \\
\hline 24 & SR11 & chr2 & 209370-209609 & 79 & 0.102 & 142 & & \\
\hline 44 & SR10 & chr2 & $382978-382817$ & 54 & 0.134 & 93 & & \\
\hline 46 & & chr2 & $391575-391309$ & 89 & 0.091 & 72 & & \\
\hline 53 & SR9 & chr2 & 614131-613982 & 50 & 0.125 & 115 & & \\
\hline 54 & SR8 & chr2 & $622941-623105$ & 55 & 0.082 & 113 & & \\
\hline $57^{\mathrm{a}, \mathrm{b}}$ & SR7 & chr2 & 684935-685219 & 94 & 0.105 & 170 & YBR233W-A & \\
\hline 68 & & chr3 & $24325-24032$ & 98 & 0.149 & 99 & YCL057C-A & removed by $S$ GD \\
\hline $82^{\mathrm{a}}$ & SR6 & chr3 & $113722-114018$ & 98 & 0.102 & 247 & YCL001W-B & \\
\hline 93 & SR5 & chr3 & $316181-315990$ & 63 & 0.121 & 161 & & \\
\hline 98 & SR4 & chr4 & $229171-229431$ & 87 & 0.175 & 38 & & \\
\hline 101 & SR3 & chr4 & $410052-409804$ & 83 & 0.151 & 68 & & \\
\hline 104 & & chr4 & $410052-409804$ & 83 & 0.108 & 77 & & \\
\hline $109^{a, b}$ & & chr4 & $603805-603587$ & 73 & 0.119 & 31 & YDR079C-A & \\
\hline 118 & SR2 & chr4 & $794716-794567$ & 50 & 0.164 & $124 / 98^{\mathrm{e}}$ & & \\
\hline 121 & SR1 & chr4 & $829143-829346$ & 67 & 0.160 & 155 & & \\
\hline 122 & & chr4 & $830646-830389$ & 86 & 0.149 & 31 & & \\
\hline 123 & & chr4 & $848063-848215$ & 51 & 0.067 & 64 & & \\
\hline 127 & & chr4 & $955124-955324$ & 67 & 0.066 & 99 & & \\
\hline $139^{a}$ & smORF8 & chr4 & $1233506-1233267$ & 79 & 0.161 & 227 & YDR379C-A & \\
\hline 144 & & chr4 & $1283788-1284033$ & 82 & 0.153 & 34 & & \\
\hline 154 & & chr9 & $96725-96522$ & 78 & 0.135 & 67 & & \\
\hline 167 & & chr5 & $59549-59740$ & 77 & 0.099 & 41 & & \\
\hline 171 & & chr5 & $117380-117183$ & 66 & 0.216 & 87 & & \\
\hline 189 & & chr5 & $318642-318806$ & 86 & 0.065 & 97 & & \\
\hline 201 & & chr6 & $48734-48925$ & 64 & 0.173 & 63 & & \\
\hline 217 & & chr7 & $93078-93308$ & 77 & 0.078 & 99 & & \\
\hline 226 & & chr7 & $418882-418700$ & 61 & 0.097 & 40 & & \\
\hline 247 & & chr7 & $733407-733622$ & 72 & 0.118 & 31 & & \\
\hline 250 & & chr7 & $974773-974573$ & 67 & 0.091 & 92 & & \\
\hline 274 & & chr8 & $280232-280393$ & 54 & 0.095 & 79 & & \\
\hline 283 & & chr8 & $453556-453705$ & 50 & 0.084 & 75 & & \\
\hline 286 & & chr8 & $467058-466954$ & 55 & 0.102 & 74 & & \\
\hline 288 & & chr8 & $466928-467110$ & 61 & 0.079 & 62 & & \\
\hline 298 & & chr10 & $181408-181250$ & 53 & 0.158 & 99 & & \\
\hline $301^{a, b}$ & & chr10 & $316419-316676$ & 86 & 0.106 & 99 & YJL062W-A & \\
\hline $303^{a}$ & & chr10 & $411124-410927$ & 66 & 0.183 & 94 & YJL012C-A & \\
\hline 313 & & chr10 & $637622-637861$ & 80 & 0.081 & 99 & & \\
\hline 318 & & chr10 & $717273-717323$ & 74 & 0.132 & 42 & & \\
\hline 324 & & chr11 & 98609-98397 & 71 & 0.186 & 25 & & \\
\hline 337 & & chr11 & $447674-447432$ & 81 & 0.156 & 27 & & \\
\hline 352 & & chr12 & $136344-136520$ & 59 & 0.197 & 85 & & \\
\hline 363 & & chr12 & $455434-455637$ & 68 & 0.105 & 34 & & \\
\hline 382 & & chr12 & $455402-455566$ & 55 & 0.078 & 99 & & \\
\hline 392 & & chr12 & $454697-455071$ & 125 & 0.170 & 98 & & \\
\hline 398 & & chr12 & $454393-454557$ & 55 & 0.059 & 99 & & \\
\hline 421 & & chr12 & $462672-462523$ & 50 & 0.045 & 99 & & \\
\hline 439 & & chr12 & $452439-452624$ & 62 & 0.086 & 99 & & \\
\hline 483 & & chr12 & 490407-490595 & 63 & 0.131 & 65 & & \\
\hline \multirow[t]{4}{*}{$494^{d}$} & SR15 & chr12 & 468959-468831 & 44 & 0.052 & 100 & & \\
\hline & & & $472611-472483$ & & & & & \\
\hline & & & $482191-482063$ & & & & & \\
\hline & & & $485843-485715$ & & & & & \\
\hline 499 & & chr12 & $582236-582006$ & 77 & 0.140 & 37 & & \\
\hline 505 & & chr12 & $708338-708168$ & 57 & 0.105 & 45 & & \\
\hline 509 & SR17 & chr12 & $760642-760355$ & 96 & 0.102 & $190 / 82^{\mathrm{e}}$ & & \\
\hline 511 & SR16 & chr12 & $815810-815983$ & 58 & 0.105 & $125 / 61^{\mathrm{e}}$ & & \\
\hline $514^{a}$ & SR18 & chr12 & $853460-853717$ & 85 & 0.282 & 230 & YLR363W-A & \\
\hline 519 & & chr12 & $950468-950262$ & 69 & 0.135 & 39 & & \\
\hline 526 & & chr13 & $167781-167623$ & 53 & 0.129 & 99 & & \\
\hline 530 & SR25 & chr13 & $337312-337602$ & 97 & 0.082 & $160 / 58^{\mathrm{e}}$ & & \\
\hline 532 & & chr13 & $550910-551134$ & 75 & 0.121 & 94 & & \\
\hline 540 & & chr13 & $733267-733455$ & 63 & 0.158 & 64 & & \\
\hline 543 & & chr13 & $804455-804691$ & 79 & 0.101 & 80 & & \\
\hline 544 & & chr13 & $887925-887731$ & 65 & 0.094 & 59 & & \\
\hline
\end{tabular}


Kessler et al.

Table 1. (Continued)

\begin{tabular}{|c|c|c|c|c|c|c|c|c|}
\hline smorf & $\begin{array}{l}\text { Original } \\
\text { name }\end{array}$ & Chromososme & Coordinates & $\begin{array}{l}\text { Size } \\
\text { (aa) }\end{array}$ & CAI & $\begin{array}{c}\text { RT-PCR } \\
\text { product (bp) }\end{array}$ & $\begin{array}{c}\text { SGD } \\
\text { annotation }\end{array}$ & Notes \\
\hline $556^{a}$ & SR22 & chr14 & $330326-330544$ & 73 & 0.130 & $170 / 37^{e}$ & YNL162W-A & \\
\hline 561 & SR21 & chr14 & $381388-381242$ & 48 & 0.102 & 90 & & \\
\hline 564 & & chr15 & $4130-4312$ & 61 & 0.121 & 55 & & \\
\hline 570 & smORF31 & chr14 & $586816-386598$ & 72 & 0.124 & 216 & & \\
\hline 577 & & chr15 & $4130-4312$ & 61 & 0.153 & 75 & & \\
\hline 580 & & chr15 & $9179-9352$ & 58 & 0.222 & 77 & & \\
\hline 590 & & chr15 & $355651-355857$ & 78 & 0.135 & 29 & & \\
\hline 591 & & chr15 & $371684-371956$ & 63 & 0.139 & 99 & & \\
\hline 598 & & chr15 & $461792-462040$ & 59 & 0.054 & 99 & & \\
\hline 601 & & chr15 & $467391-467624$ & 70 & 0.103 & 67 & & \\
\hline 625 & & chr15 & $907927-907718$ & 70 & 0.085 & 97 & & \\
\hline 626 & & chr15 & $939340-939549$ & 70 & 0.111 & 67 & & \\
\hline 631 & & chr15 & $1045189-1045344$ & 52 & 0.117 & 78 & & \\
\hline 632 & & chr15 & $1058416-1058583$ & 56 & 0.154 & 73 & & \\
\hline 640 & & chr16 & $75702-75989$ & 96 & 0.158 & 99 & & \\
\hline 643 & & chr16 & $188512-188306$ & 69 & 0.133 & 84 & & \\
\hline 655 & & chr16 & $480177-480368$ & 64 & 0.151 & 30 & & \\
\hline 667 & & chr16 & $744172-744384$ & 71 & 0.087 & 81 & & \\
\hline 672 & & chr16 & $883234-883452$ & 73 & 0.129 & 95 & & \\
\hline
\end{tabular}

This table indicates the chromosomal location based on SGD nucleotide coordinates. smORFs marked with an $\left(^{(a)}\right.$ correspond to those identified by Blandin et al. (2000). smORFs marked with a $\left({ }^{b}\right)$ sign correspond to those identified by Cliften et al. (2001). smorf19 ( $\left.{ }^{(}\right)$was originally located downstream of YBL069W (AST1), which was recently extended (Saccharomyces Genome Database, 2001.http://www.genomewww.standford.edu/Saccharomyces). smorf494 $\left({ }^{d}\right)$ is found four times in chromosome 12, downstream of the ASP3 gene in a 3.6-kb repeat (Johnston et al. 1997). For smORFs marked with ( $\left.{ }^{(}\right)$, RT-PCR was carried with two sets of primers, one set shown in Fig. 2D and a second in Fig. 2E.

started with the $S$. cerevisiae genomic sequence $(12.07 \mathrm{mb}$ total) and removed the nucleotide sequences of the previously identified 6,224 coding ORFs (Saccharomyces Genome Database, December 5 1997, http://www.genomewww.stanford.edu/Saccharomyces). We then used the remaining sequences $(3.45 \mathrm{mb})$ to identify all stop-to-stop ORFs that encode proteins with an arbitrary size of 18 amino acids or longer, based on the observation in E. coli that the majority of genes code for proteins with 18 or more amino acids (E. coli Genome Center, University of Wisconsin, Madison. http:// www.genetics.wisc.edu/).

This approach resulted in approximately 140,000 predicted ORF products, most of them shorter than 100 residues. These ORF products were next searched against a comprehensive fungal protein sequence database to identify those with potential homologs. This fungal database consists of all NCBI entries listed under "fungi" (August 20, 2000, excluding any $S$. cerevisiae sequences), plus the genomic sequences from $C$. albicans (Stanford University) and A. fumigatus (PathoGenome; http://www.LabOnWeb.com), EST sequences from $A$. nidulans, C. neoformans, F. sporotrichioides, and N. crassa (University of Oklahoma Health Sciences Center), and P. carinii EST sequences (University of Georgia). Using a cutoff score of $P \leq 10^{-4}$ (this score was chosen because it is reasonably stringent for short translated ORFs), we found 1057 S. cerevisiae predicted ORF products with potential homologs in the fungal database. ORFs were considered similar when the region of sequence similarity between the small open reading frame (smORF) and the predicted protein(s) from our database extends over the entire coding region. We then removed the ORFs which were annotated after 1997 and while this work was in progress, those that overlap with rRNA, tRNA, and Ty elements, and selected the smORFs with a start-to-stop ORF and with an upstream in-frame stop. This approach resulted in 558 smORFs that code for predicted proteins with potential fungal homologs and are located in chromosomal sections previously identified as intergenic. The 558 smORFs range in length from 18-190 codons with a mean of 64.99 codons, a median of 64 codons, and a mode of 62 codons. A unique aspect of our search is that a comprehensive database of fungal genomic and cDNA sequences was used in the sequence similarity searches as opposed to the databases containing hemiascomycetous and Saccharomyces species used in the studies of Blandin, Cliften, and coworkers (Blandin et al. 2000; Cliften et al. 2001).

\section{ORF Validation}

We chose a subset of 117 smORFs for further characterization and validation (Table 1). As a first step we determined whether smORFs were expressed in yeast cells. Primers were designed to amplify smORFs 2,8 , and 31 as well as the ACT1 gene (actin) as control (see Methods), and used for polymerase chain reaction (PCR) amplification with $S$. cerevisiae genomic DNA as a template to test the PCR amplification conditions. Products of the predicted size were obtained for all three smORFs as well as the actin control (Fig. 2A, lanes 2,6,10,14). No PCR products were obtained in reactions without the template (Fig. 2A, lanes 1,5,9,13), or using as a template RNA isolated from $S$. cerevisiae grown on rich (YPD) or complete synthetic minimal (CSM) media (Fig. 2A, lanes 3,4,7,8,11, $12,15,16)$. This indicates that these RNA samples were not contaminated with genomic DNA. We then tested for the presence of RNA transcripts originating from these smORFs as well as from the actin control, using RT-PCR. Products of the expected sizes were obtained for actin, as well as all three smORFs (Fig. 2B, lanes 2,3,5,6,8,9,11,12). This indicates that actin and the three smORFs are indeed expressed in yeast cells grown in rich and in minimal media. No RT-PCR product 
A PCR

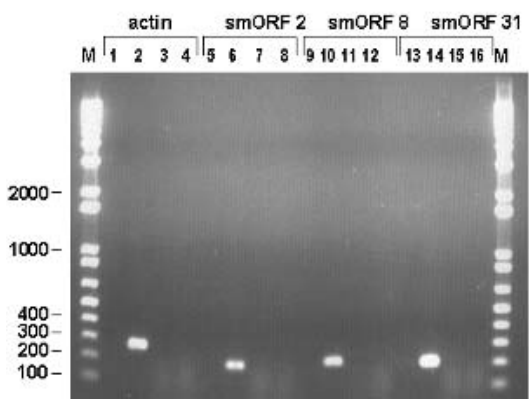

B

RT-PCR

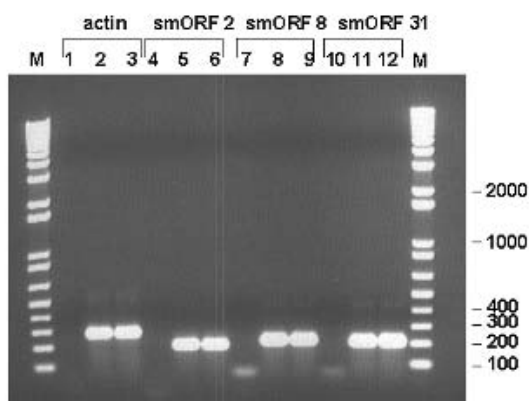

C

Two step orientation-specific RT-PCR

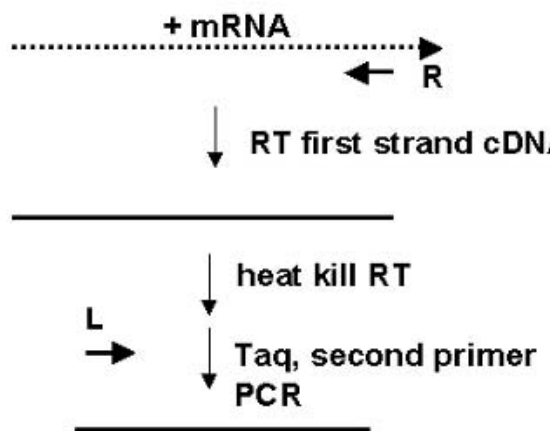

smORF2

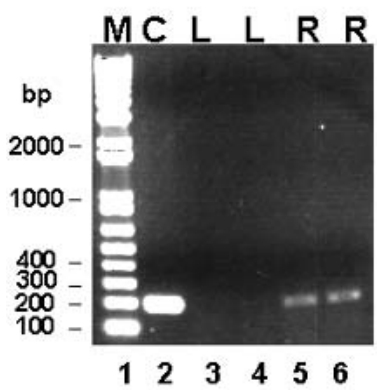

smORF8

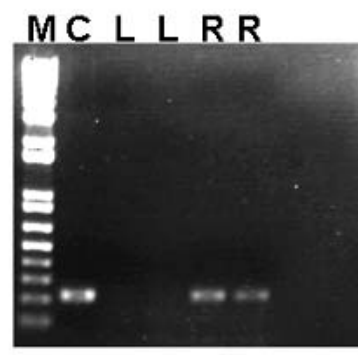

$\begin{array}{llllll}7 & 8 & 9 & 10 & 11 & 12\end{array}$

\section{smORF31}

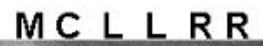

Figure 2 (Continued on next page)

was obtained in reactions without a template (Fig. 2B, lanes $1,4,7,10)$. The identity of the RT-PCR products was confirmed by cloning them, followed by restriction mapping and dideoxy sequencing (data not shown).

We then wanted to make sure that the smORF transcripts we identified were transcribed from the predicted DNA strand. To do this, we performed a variation of the RT-PCR experiment, first adding a primer complementary to the predicted mRNA and the reverse transcriptase. After first-strand cDNA synthesis, the reverse transcriptase was inactivated with heat and then Taq polymerase and both smORF-specific primers were added (Fig. 2C). Under these conditions we observed PCR products only when first-strand synthesis was conducted with primers complementary to the predicted mRNA (Fig. 2C, lanes $5,6,11,12,17,18)$. No PCR product was observed when first-strand synthesis was done with primers that have the same sequence as the mRNA (lanes $3,4,9,10,15,16)$. These results indicate that the transcripts observed for smORFs 2,8 , and 31 are made from the predicted strand.

This same study was extended to 114 additional smORFs. RT-PCR products of the expected size were obtained for 81 of these smORFs (e.g., Fig. 2D). Therefore, 84 of the 117 smORFs are transcribed from the predicted DNA strand (Table 1). The codon adaptation index (CAI, http:// www.molbio.oc.ac.uk) was calculated for the 84 smORFs (Table 1), and they range from $0.045-0.282$. Similar values were obtained for the 6224 annotated yeast genes. Nine of the smORFs in this list were recently annotated by Blandin, Cliften, and coworkers (Blandin et al. 2000; Cliften et al. 2001). We originally found SR12 downstream of the AST1gene, which was recently reannotated and extended, and now includes SR12 (Saccharomyces Genome Database, 2001; http://www.genome- www. stanford.edu/Saccharomyces). In addition we also identified smORF 68 and had an RT-PCR product for it. This smORF maps to YCL057C-A, which was recently removed from the Saccharomyces Genome Database (Saccharomyces Genome Database, 2001; http://www.genome-www.stanford.edu/ Saccharomyces). The majority of the smORFs were found only once in the budding yeast genome, except for SR15, which is present four times in chromosome 12 , downstream of $A S P 3$, in a $3.6-\mathrm{kb}$ repeat region of this chromosome (Johnston et al. 1997). Even though we detected an RT-PCR product for this SMORF, we do not know which copy was transcribed. To address the possibility that the observed smORF transcripts were products of read-through transcription from genes located upstream of the smORFs, the RT-PCR experiment was conducted using a primer complementary to the mRNA for first-strand synthesis (Fig. 2C) and with a second primer located 400 base pairs (bp) upstream of the smORF. With these conditions, no RT-PCR products were observed for 25 smORFs tested, indicating that the smORF transcripts are not the result of read-through transcription from upstream genes (data not shown). To confirm these observations, we measured the size of the transcripts coded for by smORFs 2,31 , and the $A C T 1$ gene using Northern analysis (Fig. 2E). The size of the ACT1 mRNA was 1300 nucleotides (nt; lane 3). The mRNAs for both smORF2 and 31 have a measured size of $460 \mathrm{nt}$ (Fig. 2E, lanes 5,7), in agreement with a size predicted for a 250-nt ORF, a 20-30-nt $5^{\prime}$ untranslated region (Hughes et al. 2000), a 100-nt 3' untranslated region (Graber et al. 2002), and a poly (A) tail of 70-90-nt (Hector et al. 2002). Northern analysis of smORF2 transcripts shows a band of $780 \mathrm{nt}$ that could correspond to alternative processing variants. It is unlikely that this band represents read-through from the gene located upstream of smORF2 (SNR56), because it 
D
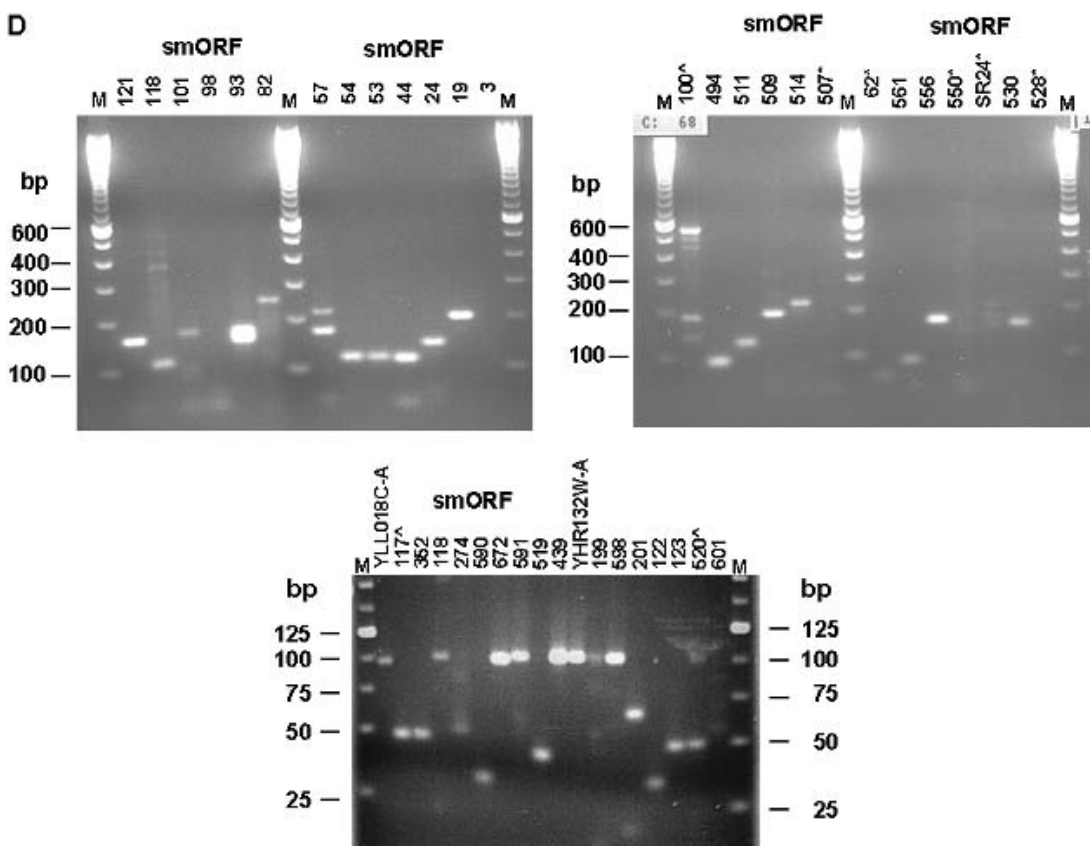

E

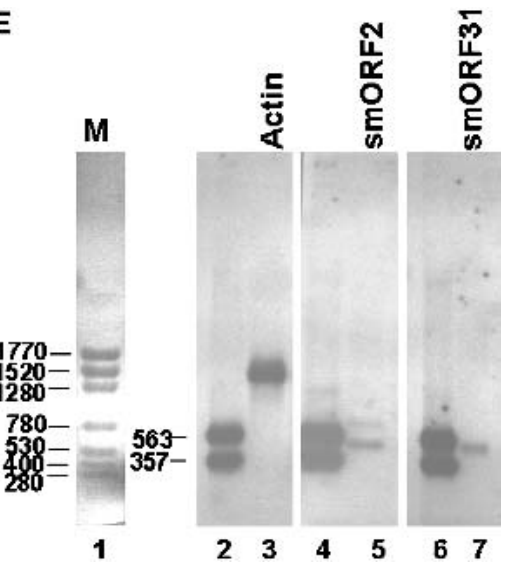

Figure 2 Experimental validation of the $S$. cerevisiae smORFs. (A) Primers specific for the yeast ACT1 gene as well as the three smORFs were used for PCR amplification using no template (lanes 1,5,9,13), 50 ng genomic DNA (lanes 2,6,10,14), 500 ng total RNA from cells grown in rich media (lanes $3,7,11,15$ ), and $500 \mathrm{ng}$ total RNA from cells grown in minimal media (lanes $4,8,12,16)$. (B) Primers specific for the yeast $A C T 1$ gene as well as the three smORFs were used for RT-PCR amplification using no template (lanes $1,4,7,10), 500 \mathrm{ng}$ total RNA from cells grown in rich media (lanes $2,5,8,11$ ), and 500 ng total RNA from cells grown in minimal media (lanes $3,6,9,12)$. PCR and RT-PCR products were fractionated on a $1 \%$ agarose gel with DNA size markers and visualized after ethidium bromide staining. Sizes of DNA fragments in bp are indicated. (C) Two-step orientation-specific RT-PCR. Primers whose sequence is complementary to the predicted mRNAs of smORF2, 8, and 31 were used for first-strand CDNA synthesis. After heat inactivation of the reverse transcriptase, PCR amplification was carried out with both smORF-specific primers (lanes 5,6,11,12,17,18). As control, the experiment was repeated using primers with the same sequence as the mRNA for first-strand CDNA synthesis (lanes $3,4,9,10,15,16)$. (D) Examples of RT-PCR results with various smORFs indicated on top. RT-PCR detection of transcripts from the annotated smORFs YLL018C-A and YHR132W-A are shown. smORFs for which RT-PCR reactions resulted in no products are indicated $\left({ }^{*}\right)$ as are those for which the product is not of the expected size $\left(^{\wedge}\right)$. These smORFs were not included in Table 1. Sizes of DNA fragments in bp are indicated. (E) Transcript size determination by Northern analysis of the ACT1 (lane 3), smORF2 (lane 5), and smORF31 (lane 7). Unlabeled RNA markers stained with methylene blue (lane 1) and labeled RNA markers (lanes 2,4,6) were fractionated together with yeast poly (A)+ RNA. Sizes are shown in nucleotides.

is located 1700 bp upstream. This results indicate that the observed transcripts originate in the promoter of the smORFs.

\section{Characterization of smORF2}

We now present a comprehensive analysis of smORF2, as homologs of this protein (smORF2p) are found in many organisms from yeast to humans (Fig. 3), and its deletion from $S$. cerevisiae exhibits a tractable phenotype. The human, Caenorhabditis elegans, Drosophila melanogaster, and $S$. pombe smORF2p homologs are about the same size as the $S$. cerevisiae counterpart. smORF2 was recently annotated by Blandin and coworkers (2000) with the systematic name YBL071W-A.

We extended our study of smORF2p to determine whether we could detect a protein product of the appropriate size. A triple HA tag was fused to the C-terminus of SmORF2 by PCR, and the wild-type smORF2 gene was replaced with the tagged version by allele replacement into the chromosome (Ederniz et al. 1997). PCR amplification of the smORF2 (HA) gene from genomic DNA, followed by cloning and sequencing, confirmed the identity of the tagged smORF2. Soluble extracts were then prepared and fractionated in $18 \%$ polyacrylamide gels containing sodium dodecyl sulfate. The proteins were transferred to a poly (vinylidene) fluoride (PVDF) membrane, and the blot was probed with anti-HA antibodies. The results show a protein band corresponding to a 9-kD protein (Fig. 4, lanes $3,4)$ in extracts prepared from cells with a tagged smORF2 gene and not in wildtype cells. This result shows that smORF2 is not only transcribed, but also encodes a detectable protein product of the expected size.

We then extended our study of smORF2 to determine whether this gene is essential or whether its deletion results in an observable phenotype. The complete smORF2 gene was deleted in a diploid yeast strain, using homologous recombination. Sporulation and tetrad analysis showed that haploid strains with a smorf $2 \Delta$ were able to grow at $30^{\circ} \mathrm{C}$ (slow growth), but not at $37^{\circ} \mathrm{C}$ (Fig. 5). To extend the notion of smORF to humans, we next tested whether the human smORF2 is a functional homolog of the yeast smORF2. The human smORF2 gene, obtained from an EST clone, and the yeast smORF2 were cloned into the pYES vector for expression in yeast under the GAL1 promoter. Clones were verified by sequencing and transformed into the smorf $2 \Delta$ strain. The resultant transformants were tested for the ability to complement the temperature-sensitive phenotype of the smorf $2 \Delta$ strain and their ability to form colonies at the restrictive temperature. The results show that the 


\section{smORF2p}

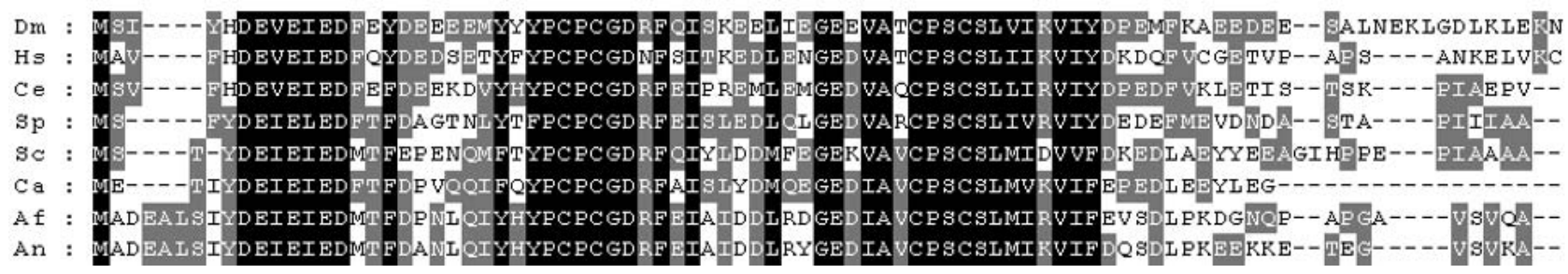

Figure 3 Multiple sequence alignments for smORF2p. smORF2p has highly conserved homologs in other fungi and in mammalian species. Abbreviations: Dm, Drosophila melanogaster; Hs, Homo sapiens; Ce, Caenorhabditis elegans; Sc, Saccharomyces cerevisiae; Ca, Candida albicans; Af, Aspergillus fumigatus; An, Aspergillus nidulans; Sp, Schizosaccharomyces pombe; Bt, Bos taurus; Mm, Mus musculus. Residues that are identical or similar in all protein homologs are shaded in black, and those identical or similar in two or more, but not all, proteins in the alignment are shaded in gray. Homology shading was done with GeneDoc (Nicholas et al. 1997).

cloned human smORF2 as well as the yeast smORF2 can complement the temperature-sensitive phenotype of the smorf $2 \Delta$ strain (Fig. 5). These results indicate that the human smORF2 is a functional ortholog of the yeast smORF2. Interestingly, the human smORF2 maps to two loci in the human genome, one in chromosome 3 where the gene contains two introns and codes for a predicted mRNA identical to the EST,

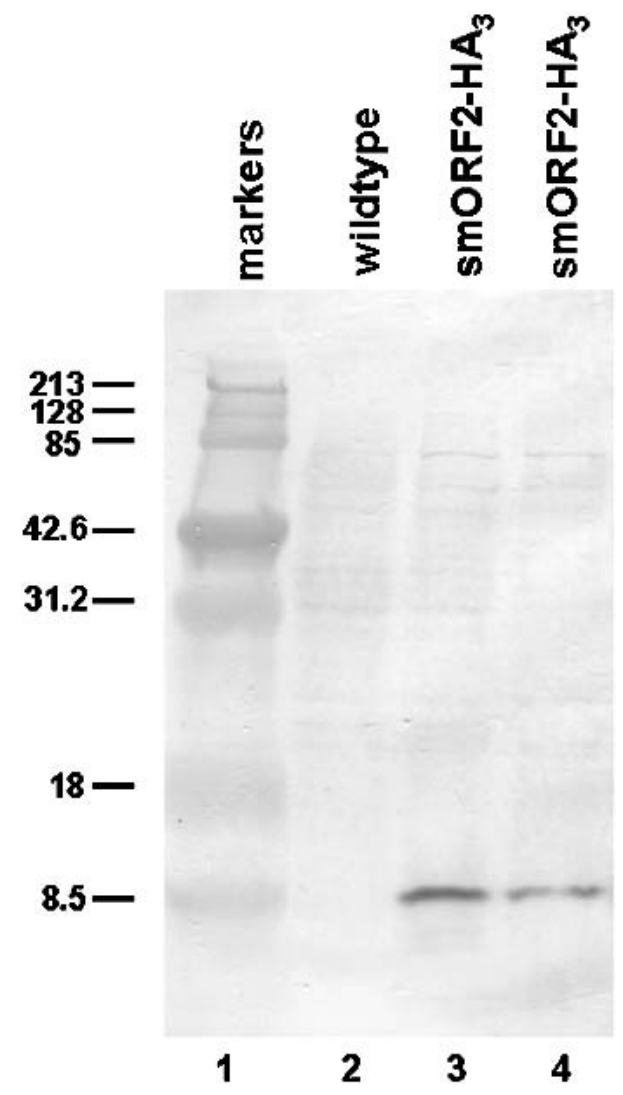

Figure 4 smORF2 is expressed in yeast. A triple HA tag was fused to the C-terminal end of smORF2 using PCR, and the wild-type smORF2 gene was replaced by the tagged smORF2 gene by allele replacement into the chromosome. Soluble extracts were prepared and analyzed in a Western blot probed with monoclonal antibodies that recognize the HA epitope. Extracts from wild-type cells (lane 2) and extracts from two separate isolates carrying the HA-tagged smORF2 (lanes $3,4)$. and to a locus in chromosome 20 without introns but with nine predicted amino acid substitutions. These data indicate that small ORFs are present and expressed in humans, and they underscore the importance of looking for small genes in the genomes of higher eukaryotes.

\section{DISCUSSION}

We have validated a method for gene identification in sequenced genomes and used it to identify new genes in $S$. cerevisiae. With this method one should be able to find new coding ORFs in $S$. cerevisiae by simply searching potential budding yeast ORF products against sequences from other fungal and nonfungal species. Even though we did not verify the expression of every predicted smORF, we found strong evidence for close to 100 new genes in the $S$. cerevisiae genome. The limited study reported here can be expanded to include smORFs that partially overlap with annotated ORFs and smORFs that are completely located within previously annotated ORFs as described recently by Kumar et al. (2002). This systematic genome comparison approach to identify ORFs will accelerate and refine genome annotation and gene identification and will impact future experimental design. The identification of conserved protein products across a wide range of species can provide us with the opportunity to use $S$. cerevisiae and other fungi to study the function of their counterparts in humans. In addition, our approach can be applied to other sequenced genomes including human in order to identify coding ORFs not readily detected by conventional methods. We anticipate finding additional smORFs which do not yet have a homolog as the amount of available sequence data increases. Conversely, we will miss some smORFs that are species-specific and therefore have no homologs in other species. We refer to these species-specific smORFs as orphan smORFs. This study and others involved in gene discovery will change the landscape of genome annotation and therefore the approach in experimental design.

\section{METHODS}

\section{RT-PCR Analysis}

Genomic DNA was prepared from strain W303 (Thomas and Rothstein 1989) using the YeaStar Genomic DNA kit (Zymo Research). Primers pairs were chosen to amplify 250-300-bp regions within the coding ORFs of the yeast ACT1 gene (5'TGTCACCAACTGGGACGATA-3'; 5'-AACCAGCGTAAATTGGAACG-3'), smORF2 (5'-TGACGAAATCGAAATCGAAG-3'; 5'-GATGCCTGCCTCTTCGTAGT-3'), smORF8 (5'-TG 


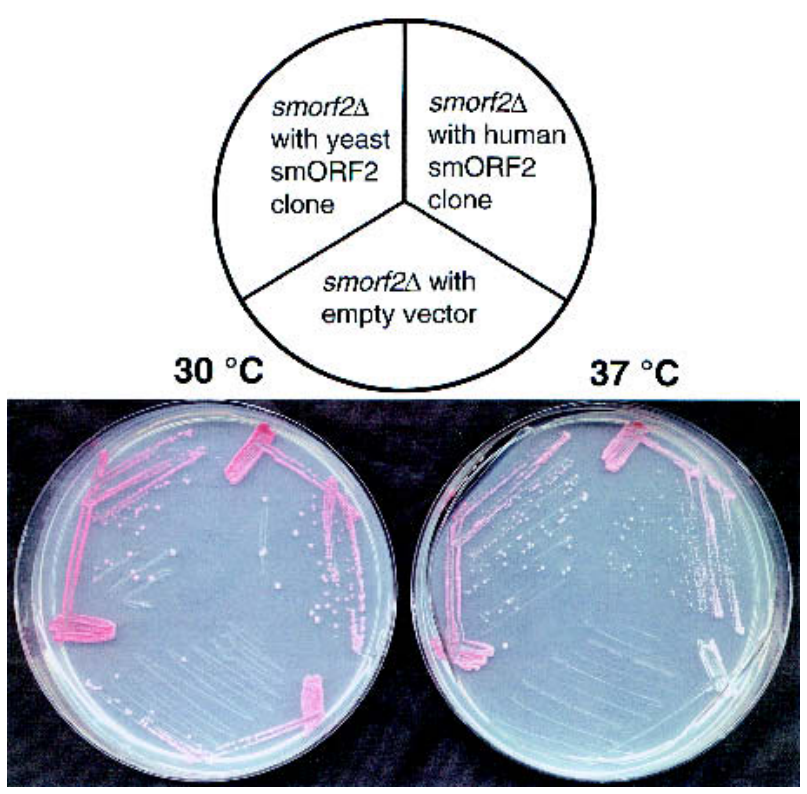

Figure 5 Human smORF2 complementation of the temperaturesensitive phenotype of the smorf $2 \Delta$ strain. A yeast strain with a deleted smORF2 (smorf $2 \Delta$ ) was transformed with plasmids carrying the wild-type yeast smORF2, human smORF2 under the control of the GAL1 promoter, or empty vector. Transformants were obtained at $30^{\circ} \mathrm{C}$, and individual colonies were streaked and then incubated at $30^{\circ} \mathrm{C}$ and $37^{\circ} \mathrm{C}$.

CCTAAGAGATTAAGTGGGTT-3'; 5'-CGTCAGTTCAGGGT GTGAAA-3'), smORF31 (5'-TGTCTGCATTATTTAATTTTC GTTC-3'; 5'-AGCTGTTAAATTGACTGATGGC-3').

RNA was isolated from $5 \times 10^{7}$ yeast cells (strain W303) growing exponentially in YEPD or synthetic complete synthetic minimal media using the RNeasy Mini kit from QIAGEN including a DNase (Roche) digestion step. RT-PCR reactions were done with the OneStep RT-PCR Kit from QIAGEN as recommended by the manufacturer. RT-PCR products were fractionated on a $1 \%$ agarose gel and visualized after ethidium bromide staining. For sequencing, the RT-PCR products were isolated from an agarose gel and then cloned into pCR2.1-TOPO (Invitrogen). To test expression of the 117 smORFs, primers were chosen within the coding sequence to amplify fragments of $25 \mathrm{bp}$ or longer.

\section{Strand-Specific RT-PCR Analysis}

First-strand ynthesis was conducted using yeast RNA, primers as indicated, and SuperScript II reverse transcriptase (Life Technologies) as recommended by the manufacturer. PCR amplification was conducted using PCR SuperMix (Life Technologies), smORF-specific primers, and $8.5 \%$ of the firststrand reaction.

For Northern analysis, $0.8 \mu \mathrm{g}$ yeast poly (A)+ RNA together with DIG-labeled and unlabeled RNA markers (Invitrogen) were fractionated in $1.5 \%$ formaldehyde-agarose gel as recommended (Ambion), transferred to Nylon membrane, and probed with single-stranded antisense probes against ACT1 (Fig. 2E, lanes 2,3), smORF2 (lanes 4,5), and smORF31 (lanes 6,7) labeled with DIG-UTP and detected as recommended (Roche).

\section{Epitope Tagging}

The modified smORF2 (HA) ${ }_{3}$ was constructed by two-way PCR. First, a PCR amplification was made using a primer corresponding to $400 \mathrm{bp}$ upstream of smORF2 (5'-
AGAAAGCCCTCAAGCTTTCCCAGCG) and a second primer containing the C-terminus of smORF2 fused to the HA tag (5'-GGAGCCTGATCCAGCGTAGTCTGGGACGTCG TA T GGGTAGCCA G CGTAGTCTGGGACGTCG T A T G G G T A G C CA G C GT A A T C C G GAACATCATAC GGGTATCCTACGGCAGCAGCGGCAATAGGCTCAGG-3'). A second amplification was carried out with a forward primer containing the tag (5'-GTAGGATACCCGTATGATGTTCCG G A T T A C G C T G G C T A C C C A T A C G A C G T C C C A G A C T A C G C T G G C T A C C C A T A C GACGT C C C A G A C T A C G C T G GA T C A G G C T C C T A A A GA T GA G A G GCTAGATCGAG-3') and a primer located downstream of smORF2 (5'-TGTCGCTTTTTCTCCTCGATGAAGC CAAGCGCCGAACCAATTGATATCATCGGCACG-3'). The tagged smORF2 gene was introduced into the smORF2 locus by allele replacement (Ederniz et al. 1997). Allele replacement was first checked by PCR and then verified by PCR amplification of the tagged gene, cloning into pCR2.1-TOPO (Invitrogen) and sequencing.

S100 extracts were prepared from diploid W303 yeast cells grown in $25 \mathrm{~mL}$ of rich medium (YPD) to mid-log phase as described (Brown et al. 1996). Immunoblots were probed with a 1:1000 dilution of the 16B12 monoclonal antibody (Berkeley Antibody) against $\mathrm{HA}_{3}$-tagged proteins.

\section{Gene Disruption}

smORF2 was disrupted in the diploid W303. Cells were transformed with a PCR fragment containing the HIS3 marker flanked by 400 bp of smORF 2 sequences. The HIS 3 sequences replaced amino acids 1 to 82 of smORF2. Histidine prototrophs were selected, and PCR was used to verify correct genomic integration. Sporulation and tetrad analysis were as described (Guthrie and Fink 1991). The human smORF2 coding sequence was amplified from I.M.A.G.E. clone 1047404 (Research Genetics). The yeast smORF2 was amplified from genomic DNA. PCR fragments were cloned into pYES2.1/V5His-TOPO (Invitrogen) and transformed into yeast as described (Guthrie and Fink 1991).

\section{ACKNOWLEDGMENTS}

We thank Kim Fechtel for valuable suggestions and for critical reading of the manuscript.

The publication costs of this article were defrayed in part by payment of page charges. This article must therefore be hereby marked "advertisement" in accordance with 18 USC section 1734 solely to indicate this fact.

\section{NOTE ADDED IN PROOF}

While this paper was in being reviewed, Fichtner and Schaffrath reported the isolation of the KTI11 gene by complementation of zymocin-resistant yeast mutants. Kti11p is smORF2. (Fichtner, L. and Schaffrath R. 2002. KTI11 and KTI13, Saccharomyces cerevisiae genes controlling sensitivity to G1 arrest induced by Kluyveromyces lactis zymocin. Mol. Microbiol. 44: $865-875$.

\section{REFERENCES}

Andrade, M.A., Daruvar, A., Casari, G., Schneider, R., Termier, M., and Sander, C. 1997. Characterization of new proteins found by analysis of short open reading frames from the full yeast genome. Yeast 13: 1363-1374.

Basrai, M.A., Velculescu, V.E., Kinzler, K.W., and Hieter, P. 1999. NORF5/HUG1 is a component of the MEC1-mediated checkpoint response to DNA damage and replication arrest in Saccharomyces cerevisiae. Mol. Cell. Biol. 19: 7041-7049.

Blandin, G., Durrens, P., Tekaia, F., Aigle, M., Bolotin-Fukuhara, M., Bon, E., Casaregola, S., de Montigny, J., Gaillardin, C., Lepingle, A., et al. 2000. Genomic exploration of the hemiascomycetous yeasts: 4 . The genome of Saccharomyces cerevisiae revisited. FEBS Lett. 487: 31-36. 
Brown, C.E., Tarun Jr., S.Z., Boeck, R., and Sachs, A. 1996. PAN3 encodes a subunit of the Pab1p-dependent poly(A) nuclease in Saccharomyces cerevisiae. Mol. Cell. Biol 16: 5744-5753.

Cliften, P.F., Hillier, L.W., Fulton, L., Graves, T., Miner, T., Gish, W.R., Waterston, R.H., and Johnston, M. 2001. Surveying Saccharomyces genomes to identify functional elements by comparative DNA sequence analysis. Genome Res. 11: 1175-1186.

Erdeniz, N., Mortensen, U.H., and Rothstein, R. 1997. Cloning-free PCR-based allele replacement methods. Genome Res. 7: 1174-1183.

Goffeau, A., Barrell, B.G., Bussey, H., Davis, R.W., Dujon, B., Feldmann, H., Galibert, F., Hoheisel, J.D., Jacq, C., Johnston, M. et al. 1996. Life with 6000 genes. Science 274: 546-567.

Gopal, S., Schroeder, M., Pieper, U., Sczyrba, A., Aytekin-Kurban, G., Bekiranov, S., Fajardo, J.E., Eswar, N., Sanchez, R., Sali, A., et al. 2001. Homology-based annotation yields 1,042 new candidate genes in the Drosophila melanogaster genome. Nat. Genet. 27: $337-340$.

Graber, J.H., McAllister, G.D., and Smith, T.F. 2002. Probabilistic prediction of Saccharomyces cerevisiae mRNA 3'-processing sites. Nucleic Acids Res. 30: 1851-1858.

Guthrie, C., Fink, G.R., Simon, M.I., and Abelson, J.N., Eds. 1991. Guide to yeast genetics and molecular biology. Methods Enzymol. Vol. 194. Academic Press, New York, NY.

Hector, R.E., Nykamp, K.R., Dheur, S., Anderson, J.T., Non, P.J., Urbinati, C.R., Wilson, S.M., Minvielle-Sebastia, L., and Swanson, M.S. 2002. Dual requirement for yeast hnRNP Nab2p in mRNA poly(A) tail length control and nuclear export. EMBO J. 21: $1800-1810$

Hughes, J.D., Estep, P.W., Tavazoie, S., and Church, G.M. 2000. Computational identification of cis-regulatory elements associated with groups of functionally related genes in Saccharomyces cerevisiae. J. Mol. Biol. 296: 1205-1214.

Johnston, M., Hillier, L., Riles, L., Albermann, K., Andre, B., Ansorge, W., Benes, V., Bruckner, M., Delius, H., Dubois, E., et al. 1997. The nucleotide sequence of Saccharomyces cerevisiae chromosome XII. Nature 387: 87-90.

Kumar, A., Harrison, P.M., Cheung, K.H., Lan, N., Echols, N., Bertone, P., Miller, P., Gerstein, M.B., and Snyder, M. 2002. An integrated approach for finding overlooked genes in yeast. Nat. Biotechnol. 20: 58-63.

Kupfer, D.M., Reece, C.A., Clifton, S.W., Roe, B.A, and Prade, R.A. 1997. Multicellular ascomycetous fungal genomes contain more than 8000 genes. Fungal Genet. Biol. 21: 364-372.

Mackiewicz, P., Kowalczuk, M., Mackiewicz, D., Nowicka, A., Dudkiewicz, M., Laszkiewicz, A., Dudek, M.R., and Cebrat, S. 2002. How many protein-coding genes are there in the Saccharomyces cerevisiae genome? Yeast 19: 619-629.

Mewes, H.W., Albermann, K., Bahr, M., Frishman, D., Gleissner, A., Hani, J., Heumann, K., Kleine, K., Maierl, A., Oliver, S.G., et al.
1997. Overview of the yeast genome. Nature 387: 7-65. Nicholas, K.B., Nicholas Jr., H.B., and Deerfield II, D.W. 1997. GeneDoc: Analysis and visualization of genetic variation. EMBnet News 4: 14-17. http://www.no.embnet.org/embnet.news/vol4_2/ contents.html

Olivas, W.M., Muhlrad, D., and Parker, R. 1997. Analysis of the yeast genome: Identification of new noncoding and small ORF-containing RNAs. Nucleic Acids Res. 25: 4619-4625.

Reboul, J., Vaglio, P., Tzellas, N., Thierry-Mieg, N., Moore, T., Jackson, C., Shin-I, T., Kohara, Y., Thierry-Mieg, D., Thierry-Mieg, J., et al. 2001. Open-reading-frame sequence tags (OSTs) support the existence of at least 17,300 genes in $C$. elegans. Nat. Genet. 27: 332-336.

Ross-Macdonald, P., Coelho, P.S., Roemer, T., Agarwal, S., Kumar, A., Jansen, R., Cheung, K.H., Sheehan, A., Symoniatis, D., Umansky, L., et al. 1999. Large-scale analysis of the yeast genome by transposon tagging and gene disruption. Nature 402: 413-418.

Thomas, B.J. and Rothstein, R. 1989. The genetic control of direct-repeat recombination in Saccharomyces: The effect of rad52 and rad1 on mitotic recombination at GAL10, a transcriptionally regulated gene. Genetics 123: 725-738.

Tzung, K.W., Williams, R.M., Scherer, S., Federspiel, N., Jones, T., Hansen, N., Bivolarevic, V., Huizar, L., Komp, C., Surzycki,. R., et al. 2001. Genomic evidence for a complete sexual cycle in Candida albicans. Proc. Natl. Acad. Sci. 98: 3249-3253.

Velculescu, V.E., Zhang, L., Zhou,. W., Vogelstein, J., Basrai, M.A., Bassett Jr., D.E. Hieter, P., Vogelstein, B., and Kinzler, K.W. 1997. Characterization of the yeast transcriptome. Cell 88: 243-251.

Winzeler, E.A. and Davis, R.W. 1997. Functional analysis of the yeast genome. Curr. Opin. Genet. Dev. 7: 771-776.

Zeng, Q., Morales, A., and Cottarel, G. 2001. Fungi and humans: Closer than you think. Trends Genet. 17: 682-684.

\section{WEB SITE REFERENCES}

http://www.LabOnWeb.com; Aspergillus fumigatus genomic sequences are available on the Web.

http://www.genetics.wisc.edu/; Escherichia coli Genome Center. October 13, 1998, revision date: E. coli Genome Center, University of Wisconsin, Madison.

http://genome-www.stanford.edu/Saccharomyces/; Saccharomyces Genome Database as of December 51997.

http://genome-www.stanford.edu/Saccharomyces/; Saccharomyces Genome Database as of October 2001.

http://www.molbio.oc.ac.uk; Codon adaptation index (CAI).

Received March 1, 2002; accepted in revised form November 7, 2002.
Genome Research www.genome.org 
Genome Research 13: 264-271 (2003)

Systematic Discovery of New Genes in the Saccharomyces cerevisiae Genome

Marco M. Kessler, Qiandong Zeng, Sarah Hogan, Robin Cook, Arturo J. Morales, and

Guillaume Cottarel

The affiliations in the above article were printed incorrectly. Below please find the correct affiliations for the authors.

Marco M. Kessler, ${ }^{1}$ Qiandong Zeng, ${ }^{2}$ Sarah Hogan, ${ }^{5}$ Robin Cook, ${ }^{3}$ Arturo J. Morales,, 6 and Guillaume Cottarel $^{4}$

${ }^{1}$ Microbia, Inc. Cambridge, MA 02141, USA; ${ }^{2}$ GeneData (USA), Inc. Waltham, MA 02451, USA; ${ }^{3}$ Department of Biology, University of Maryland, College Park, MD 20742, USA; ${ }^{4}$ Dana Farber Cancer Institute and Department of Genetics, Harvard Medical School, Boston, MA 02115, USA; ${ }^{5}$ Genome Therapeutics Corporation, Waltham, Massachusetts 02453, USA

${ }^{6}$ Corresponding author.

E-MAIL arturo.morales@genomecorp.com; FAX (781) 398-2476.

Genome Research 13: 485-491 (2003)

The Development of a Highly Informative Mouse Simple Sequence Length Polymorphism (SSLP) Marker Set and Construction of a Mouse Family Tree Using Parsimony Analysis

Philip D. Witmer, Kimberly F. Doheny, Marcia K. Adams, Corinne D. Boehm, Jane S. Dizon, Janet L. Goldstein, Tira M. Templeton, Ariana M. Wheaton, Penny N. Dong, Elizabeth W. Pugh, Robert L. Nussbaum, Kent Hunter, Jennifer A. Kelmenson, Lucy B. Rowe, and Michael J. Brownstein

Tira M. Templeton's name was spelled incorrectly in the above article. It should be Tiva M. Templeton. 


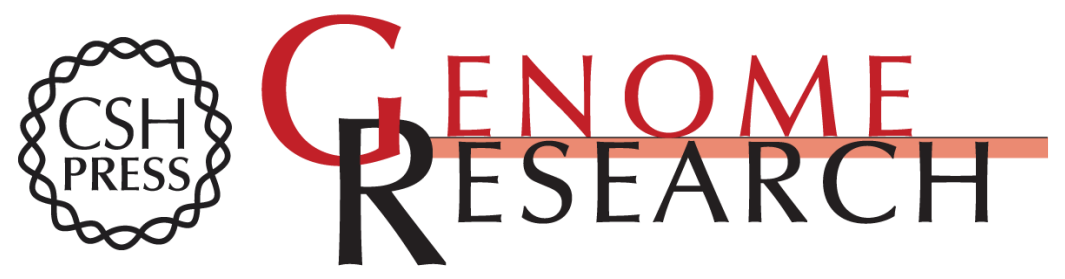

\section{Systematic Discovery of New Genes in the Saccharomyces cerevisiae Genome}

Marco M. Kessler, Qiandong Zeng, Sarah Hogan, et al.

Genome Res. 2003 13: 264-271

Access the most recent version at doi:10.1101/gr.232903

Related Content Erratum for vol. 13, p. 264

Genome Res. May, 2003 13: 1027

References This article cites 25 articles, 8 of which can be accessed free at:

http://genome.cshlp.org/content/13/2/264.full.html\#ref-list-1

Articles cited in:

http://genome.cshlp.org/content/13/2/264.full.html\#related-urls

\section{License}

Email Alerting Receive free email alerts when new articles cite this article - sign up in the box at the Service top right corner of the article or click here.

\section{Affordable, Accurate Sequencing.}

To subscribe to Genome Research go to:

https://genome.cshlp.org/subscriptions 